\title{
Imaging structural brain development in childhood and adolescence
}

\author{
Christian K. Tamnes \\ University of Oslo, Oslo, Norway \\ Kathryn L. Mills \\ University of Oregon, Oregon, USA
}

Corresponding Author:

Kathryn L. Mills

University of Oregon

115 Lewis Integrative Sciences

Eugene, OR 97403

USA

+15023772362

kathryn.1.mills@gmail.com

This is a preprint of the following book chapter:

Tamnes CK, Mills KL (2020). Imaging structural brain development in childhood and adolescence. In D Poeppel, GR Mangun, MS Gazzaniga (eds.), The Cognitive Neurosciences VI (pp.17-25). MIT Press.

Acknowledgements:

We thank Nandita Vijayakumar and Lara M. Wierenga for comments on earlier drafts of the manuscript. Christian K. Tamnes is funded by the Research Council of Norway and Kathryn L. Mills is funded by the National Institutes of Health R01 MH107418. 


\begin{abstract}
The human brain undergoes a remarkably protracted development. Magnetic resonance imaging (MRI) has allowed us to capture these changes through longitudinal investigations. In this chapter, we describe the typical developmental trajectories of human brain structure between childhood and early adulthood. We focus on measurements of brain morphometry and measurements derived from diffusion tensor imaging (DTI). By integrating findings from multiple longitudinal investigations with seminal cellular studies, we describe neurotypical patterns of structural brain development and possible underlying biological mechanisms. Finally, we highlight several new measures and approaches to examine structural brain development.
\end{abstract}




\section{Introduction}

Since the 1990s, there have been several longitudinal investigations that have examined brain development using Magnetic Resonance Imaging (MRI). Through these studies we have learned that the human brain undergoes a particularly protracted development, with some aspects of our brain maturing into the third decade of life. This chapter will review the current literature on the development of brain structure as measured through MRI. We will focus on aspects of brain morphometry, as well as tissue microstructure as measured through diffusion tensor imaging (DTI). We will then discuss biological mechanisms underlying developmental changes in brain structure and highlight new imaging and analytic approaches to the study of structural brain development. While there has been a recent concerted effort to understand aspects of brain development during infancy and early childhood using MRI, we will focus primarily on brain development during later childhood and adolescence.

\section{Brain Structural and Microstructural Development}

MRI is an imaging technique based on the principles of nuclear magnetic resonance that detects proton signals from water molecules and that allows us to produce high quality images of the internal structure of living organs. MRI protocols designed to create anatomical images of the brain rely on signal intensities and contrasts to distinguish between grey matter, white matter, and cerebrospinal fluid (CSF), while other protocols can create e.g. images to probe tissue microstructural properties (Figure 1).

\section{Global volumes}

It is important to note that the cranial cavity itself continues to grow into the second decade of life. An investigation of four longitudinal developmental datasets presented evidence that intracranial volume increases around $1 \%$ annually between late childhood and mid-adolescence, when it begins to stabilize (Mills et al., 2016). In this regard, the growth of intracranial volume resembles the growth trajectories of other physical measures such as height and bone density, although changes in body growth do not fully account for changes in intracranial volume. In contrast to the steady increase in intracranial volume into mid-adolescence, whole brain volume (the sum of grey and white matter) reduces in size during adolescence before stabilizing in the early twenties (Mills et al., 2016). When these findings are considered alongside those from a 
large meta-analysis of longitudinal studies, it appears that whole brain volume increases until around age 13 years, then decreases until some point in the early twenties, after which it remains relatively stable until around age 40, when it begins to decrease again (Hedman, van Haren, Schnack, Kahn, \& Hulshoff Pol, 2012). These findings go beyond early assertions that the brain was close to adult volume by early childhood, as it is now clear that the overall size of the human brain continue to change across the first two decades of life.

Critically, volumetric growth of the two main subcomponents of the brain, grey matter and white matter, follow distinct developmental trajectories. Grey matter, i.e. the cerebral and cerebellar cortex and distinct subcortical structures, is composed of neuronal bodies, glial cells, dendrites, blood vessels, extracellular space and both unmyelinated and myelinated axons. Cortical grey matter increases rapidly after birth, approximately doubling in volume in the first year of life (Gilmore et al., 2012). It then reaches its greatest volume in childhood, and begins to decrease in late childhood and throughout adolescence, before stabilizing in the third decade of life (Lebel \& Beaulieu, 2011; Mills et al., 2016). In a study of four longitudinal datasets, we calculated that cortical volume decreases by (on average) 1.4\% annually between late childhood and early adulthood, with the sharpest decline in volume occurring in early-to-mid adolescence (Tamnes, Herting, et al., 2017). In contrast, cerebral white matter, which occupies almost half of the human brain and consists largely of organized myelinated axons, continues to increase in volume into at least the second decade of life, but begins to decelerate at some point in mid-to-late adolescence (Lebel \& Beaulieu, 2011; Mills et al., 2016). In addition to these tissue specific patterns, there are also component specific and regional differences in brain developmental timing and pace, which have been linked to adolescent specific changes in behavior.

\section{Cerebral cortex}

Because the cerebral cortex is a layer of tissue enveloping the cerebrum, it is often measured in terms of thickness, surface area, or their product, volume. Cortical thickness and surface area are influenced by different evolutionary, genetic, and cellular processes, and show unique developmental changes (Mills, Lalonde, Clasen, Giedd, \& Blakemore, 2014; Tamnes, Herting, et al., 2017; Vijayakumar et al., 2016; Wierenga, Langen, Oranje, \& Durston, 2014). While there is 
overall substantial variability in brain size in both mature and developing humans, there is less inter-individual variability in cortical thickness than in surface area.

Average cortical thickness follows a similar nonlinear decreasing trajectory as cortical volume (around 1\% annually), although the decline in average cortical thickness is more pronounced across the second decade of life before flattening out around age 20 (Tamnes, Herting, et al., 2017). Cortical thickness begins to decrease much earlier than grey matter volume or cortical surface area, with this process observed as early as at 4 years of age (Walhovd, Fjell, Giedd, Dale, \& Brown, 2016). In contrast, total cortical surface area increases in early development and begins to decrease in an almost linear fashion (around $0.5 \%$ annually) from late childhood to early adulthood (Tamnes, Herting, et al., 2017).

The cerebral cortex does not develop uniformly. Investigations of structural brain development starting in middle childhood have consistently found decelerating change in posterior cortical regions and accelerating change in anterior regions, in line with the posterior-anterior theory of cortical maturation (Yakovlev \& Lecours, 1967). For example, the parietal lobes and lateral occipital cortices (involved in sensory processing) show larger volumetric reductions in late childhood and early adolescence, whereas the medial frontal cortex and anterior temporal cortex pick up the pace in the teen years (Tamnes et al., 2013). The more pronounced changes in brain structure that occur during the second decade of life are likely related to cognitive processes involved the developmental tasks of this period of life.

Notably, not all cortical regions undergo significant macrostructural changes between late childhood and early adulthood. Studies of several longitudinal datasets have found evidence for little to no change in the central sulcus, medial temporal, and medial occipital cortices (Mutlu et al., 2013; Tamnes et al., 2013). Given that the central sulcus and medial occipital cortices are involved in primary sensory processes, they likely undergo more rapid change at earlier ages. Certain cortical regions also show relatively greater surface area expansion between childhood and young adulthood (Hill et al., 2010). Between ages 4-20 years, this includes the lateral and medial temporal, cingulate, lateral orbitofrontal, superior and inferior frontal, insular, temporoparietal, cuneus, and lingual cortices (Fjell et al., 2015). 


\section{Cortical topography}

The human cortex is highly convoluted, with approximately one third of the cortical surface exposed on gyri, and two-thirds buried within sulci. The gyrification index of the whole brain is defined as the ratio of the total folded cortical surface over the total perimeter of the brain (Zilles, Armstrong, Schleicher, \& Kretschmann, 1988), whereas the local gyrification index measures the degree of cortical folding at specific points of the cortical surface (Schaer et al., 2008). The gyrification index of the human brain decreases between childhood and young adulthood, whereas the amount of exposed cortical surface increases from childhood to late adolescence (Alemán-Gómez et al., 2013; Raznahan, Shaw, et al., 2011). One longitudinal study demonstrated that the cortex "flattens" during adolescence, mostly due to decreases in sulcal depth and increases in sulcal width (Alemán-Gómez et al., 2013). The developmental changes in

local gyrification varies across the cortex, with regions in medial prefrontal cortex, occipital cortex, and temporal cortex undergoing little to no change between ages 6-30 years (Mutlu et al., 2013). However, similar to what has been found in whole brain (Raznahan, Shaw, et al., 2011) and lobar-level (Alemán-Gómez et al., 2013) analyses, Mutlu et al. (2013) observed linear decreases in local gyrification index across the majority of the cortex.

\section{Subcortical structures}

Several subcortical structures and cortical infolds show substantial structural change between childhood and young adulthood, although generally at a lower rate than is observed in the cortex (Tamnes et al., 2013). Longitudinal studies have found that the thalamus, pallidum, amygdala, caudate, putamen, and nucleus accumbens all show significant changes in volume across the second decade of life (Goddings et al., 2014; Herting et al., 2018; Wierenga, Langen, Ambrosino, et al., 2014). The caudate, putamen and nucleus accumbens undergo linear reductions during this time, whereas the amygdala, thalamus and pallidum follow non-linear increases. These findings are in contradiction with hypotheses and developmental models that assume that subcortical structures are mature by adolescence, as it is now clear that subcortical regions are still undergoing structural development throughout the second decade of life.

\section{White matter microstructure}


Diffusion MRI (dMRI) has over the last two decades grown in popularity as a method to study brain development, particularly the development of white matter. dMRI uses the phenomenon of natural movement of water molecules in the brain to indirectly obtain information about the underlying tissue microstructure (Le Bihan \& Johansen-Berg, 2012). This is possible since water diffusion in biological tissue is not free and uniform (isotropic), but reflects interactions with obstacles, such as cell membranes and myelin, and is therefore not necessarily the same in all direction (anisotropic). The diffusion patterns can reveal details about tissue architecture at a micrometer scale well beyond the usual millimetric resolution of morphometric MRI.

Typical quantification of dMRI is achieved in a tensor model and this is referred to as DTI. Several indices can be derived; Fractional anisotropy (FA) is used as a measure of the directionality of diffusion, mean diffusivity (MD) reflects the overall magnitude of diffusion, and axial (AD) and radial diffusivity (RD) are diffusivity along and across the longest axis of the diffusion tensor, respectively. These indices can be analyzed on a voxel-wise basis or in regions of interest. Tractography techniques can be used to reconstruct long-range connections, yielding possibilities for inferring patterns of structural connectivity (Jbabdi \& Behrens, 2013). However, the current techniques also have known limitations (see e.g. Maier-Hein et al., 2017).

The major white matter fiber pathways in the brain are present and identifiable at birth, but very rapid changes in DTI indices are seen across infancy (Qiu, Mori, \& Miller, 2015). For example, a large longitudinal study of young children indicated that in the first two years of life, FA in ten major tracts increases by $16-55 \%$, RD decreases by $24-46 \%$, and AD decreases by $13-28 \%$, with faster changes in the first year than in the second for all tracts investigated (Geng et al., 2012). Such massive changes are perhaps not surprising given the enormous behavioral and psychological development in this period of life.

As for later childhood and adolescence, many cross-sectional and an increasing number of longitudinal DTI studies document consistent patterns of continued development of white matter microstructure. With increasing age, FA increases, while MD and RD decreases, in most white matter regions, but the results for $\mathrm{AD}$ are less consistent (Tamnes, Roalf, Goddings, \& Lebel, 2017a). For example, Krogsrud et al. (2016) focused on the preschool and early school years, 
and found that for most white matter regions, FA showed linear increase over time, while MD and RD showed linear decrease. Lebel and Beaulieu (2011) studied a much broader age-range, 532 years, and used tractography for ten major white matter tract. Almost all showed nonlinear developmental trajectories, with decelerating increases for FA and decelerating decreases for MD, primarily due to decreasing RD (see also Simmonds, Hallquist, Asato, \& Luna, 2014).

The timing and rates of the DTI developmental changes vary regionally in the brain. A pattern of maturation in which major tracts with frontotemporal connections develop more slowly than other tracts has emerged (Lebel, Walker, Leemans, Phillips, \& Beaulieu, 2008). Lebel and Beaulieu (2011) also found a pattern in which changes in DTI parameters were mostly complete by late adolescence for projection and commissural tracts, while post-adolescent development was indicated for both FA and MD in association tracts. Of the major fiber bundles, the cingulum, implicated in e.g. cognitive control, and the uncinate fasciculus, implicated in emotion and episodic memory, are among those that have been shown to have particularly prolonged development (Lebel et al., 2012; Olson, Heide, Alm, \& Vyas, 2015).

\section{Relating structural brain development to biological development}

While most studies assess human brain development in relation to chronological age, there are other developmental processes that occur during the first two decades of life which likely impact on brain development, such as body growth and puberty. There are several reasons why age might not be the most appropriate measure against which to judge brain development. For one, age only explains a certain proportion of the variance in modeled trajectories. Further, age provides little information about the possible cellular and molecular mechanisms underlying observed changes. During late childhood and adolescence, individuals undergo physical changes such as a height growth spurt and puberty, which happen at different ages across individuals, and on average at different times for girls and boys.

\section{Sex differences}

Although males on average show larger global and regional brain volumes than females and sex differences have been reported for many other imaging measures (Ruigrok et al., 2014), findings are much less clear for sex differences in developmental changes and trajectories across 
childhood and adolescence (Herting et al., 2017; Mutlu et al., 2013; Vijayakumar et al., 2016). It has also proven difficult to clearly describe how puberty and related hormonal changes affect brain structural and microstructural development, and there are few longitudinal studies (Herting \& Sowell, 2017). However, one large longitudinal study found that age and pubertal development had both independent and interactive influences on volume for the amygdala, hippocampus and putamen in both sexes, and the caudate in females (Goddings et al., 2014). The relatively subtle (or inconclusive) evidence for mean sex differences in brain development, might suggest that we need to move beyond mean level differences. Robust sex differences in variability in brain measures have recently been shown in both developmental (Wierenga, Sexton, Laake, Giedd, \& Tamnes, n.d.) and adult samples (Ritchie et al., 2017), with males showing greater variance expressed at both upper and lower extremities of the distributions, something which might have functional and clinical implications.

\section{Cellular and molecular mechanisms underlying structural changes}

What do developmental changes, as assessed by e.g. T1-weighted MRI or DTI scans, reflect on a cellular and molecular level? To date, studies that have directly tested these relationships in humans are scarce. However, there are several hypotheses concerning the mechanisms underlying these observed developmental changes (Paus, 2013). One hypothesis is that reductions in grey matter volume during adolescence partly reflect synaptic pruning. However, synaptic boutons are very small and comprise only a fraction of grey matter volume. Even when synapses are particularly dense, they are estimated to represent only $2 \%$ of a cubic millimeter of neuropil or less than $1.5 \%$ cortical volume (Bourgeois \& Rakic, 1993). Given this small percentage, it is unlikely that the marked decreases in cortical volume observed across adolescence are mainly reflective of synaptic pruning. The reduction in number of synapses might, however, in addition to a reduction in neuropil, also be accompanied by a reduction in the number of cortical glial cells or other processes. These events could together account for more of the cortical structural changes observed during development, although this remains a speculation. Encroachment of subcortical white matter, and/or continued intracortical myelination, likely impact on measurements of cortical grey matter, by changing the signal intensity values and contrasts such that the boundary between white and grey matter is moved outwards with increasing age. Undoubtedly, there is a myriad of both parallel and interacting neurobiological 
processes underlying the macrostructural changes observed during childhood and adolescence in MRI studies.

Similarly, many factors, including axon caliber, myelin content, fiber density, water content, crossing or diverging fibers and partial voluming, influence DTI indices (Beaulieu, 2009). Developmental changes in DTI indices are thought to mainly relate to increasing axon caliber and continued myelination, as well as changes in fiber packing density (Paus, 2010). Animal studies indicate that axonal membranes are the primary determinants of FA, while myelin has a modulating role (Beaulieu, 2009; Concha, Livy, Beaulieu, Wheatley, \& Gross, 2010). For example, rodent dysmyelination models show that FA values still indicate anisotropy and reduce only by $\sim 15 \%$ in the complete absence of myelin (Beaulieu, 2009). Further, a rare study comparing human in vivo DTI with subsequent microscopy in patients with epilepsy found a robust positive correlation between FA and axonal membranes (Concha et al., 2010). Animal studies do however also consistently indicate that RD is particularly sensitive to de- and dysmyelination (e.g. Song et al., 2005), and correlations between DTI and myelin content and to a lesser degree axon count have also been shown in postmortem human brains of patients with multiple sclerosis (Schmierer et al., 2007). The myelin content interpretation has because of these and other findings often been stressed. Although myelination, a process that begins between weeks 20 and 28 of gestation, has been shown to continue throughout childhood and adolescence (Benes, 1989; Benes, Turtle, Khan, \& Farol, 1994; Yakovlev \& Lecours, 1967), it does not logically follow from these rodent and postmortem studies that healthy developmental changes in RD in humans reliably indicates myelination (Paus, 2010). DTI parameters are sensitive to general diffusion properties of brain tissue and are not selective markers of specific biological properties.

The relative roles of specific cellular and molecular processes for developmental changes in brain structure and microstructure are likely also age-dependent, with different contributions for instance in infancy, during adolescence and in old age. Precise interpretations of the underlying mechanisms of morphometric or DTI developmental changes are thus challenging and should be done with great caution. However, investigating multiple imaging indices concurrently might yield additional information to better characterize tissue properties, and new imaging techniques, 
as well as studies combining imaging and histology, can hopefully increase our understanding of the cellular and molecular developmental processes.

\section{Future Directions}

Beyond well-established morphometric approaches and DTI, imaging acquisition and analysis techniques are ever evolving, promising to provide more sensitive or specific measures. In this section, we briefly present a few selected emerging imaging and analytic approaches, and discuss their application to structural brain development in childhood and adolescence.

A small, but increasing number of studies use surface-based methods and examine age-related differences in specific signal intensity contrasts, such as cortical gray/white matter contrast (Lewis, Evans, \& Tohka, 2017) or the T1-weighted/T2-weighted ratio, also referred to as cortical "myelin mapping" (Glasser \& Van Essen, 2011; Grydeland, Walhovd, Tamnes, Westlye, \& Fjell, 2013) (see also (Geeraert et al., (2017) for a comparison of other neuroimaging markers of myelin content in children). In relation to the more widely used measures, these approaches appear to provide partly independent and possibly more specific biomarkers of brain structural alterations in development, but longitudinal developmental studies are not yet available.

More recent and advanced dMRI methods compared to DTI, including diffusion kurtosis imaging (DKI) and neurite orientation dispersion and density imaging (NODDI), also aim to provide biologically more specific measures. Developmental studies using these methods are becoming more common, yet only cross-sectional studies are so far available (Tamnes, Roalf, Goddings, \& Lebel, 2017b). NODDI studies indicate that FA increase during childhood and adolescence is dominated by increasing neurite density index (NDI), which point to increasing myelin and/or axonal packing, but negligible changes in axon coherence during development (Chang et al., 2015; Mah, Geeraert, \& Lebel, 2017). Moreover, results indicated that NODDI metrics predicted chronological age better than DTI metrics (Chang et al., 2015; Genc, Malpas, Holland, Beare, \& Silk, 2017). Initial applications of these methods thus demonstrate utility in studying brain development. However, they currently require relatively long scan times, a hurdle for developmental studies. 
An increasingly popular analytic approach is structural covariance, which refers to correlations across individuals in the properties of pairs of brain regions, and aims to inform us on structural connectivity (Alexander-Bloch, Giedd, \& Bullmore, 2013). A few longitudinal studies have used the approach of "maturational coupling", i.e. covariance in longitudinal changes across subjects. It has been found that fronto-temporal association cortices show the strongest and most widespread maturational coupling with other cortical areas, while lower-order sensory cortices show the least (Raznahan, Lerch, et al., 2011). Another study looked at cortico-subcortical structural change relationships and found that these partly correspond to known functional networks, for example, longitudinal change in hippocampal volume was found to be associated with longitudinal changes in cortical areas involved in episodic memory (Walhovd et al., 2015). It has also been indicated that maturational covariance, presumably reflecting coordinated development between brain regions, may be responsible for cross-sectional structural covariance (Alexander-Bloch, Raznahan, Bullmore, \& Giedd, 2013). Finally, a recent study indicates links between verbal intelligence and the strength of structural couplings of cortical regions in children and adolescents (Khundrakpam et al., 2017).

Beyond these measures, graph theoretical analyses are opening up new perspectives on the development of brain networks, potentially across imaging modalities and scales (Betzel \& Bassett, 2017). Although many features of complex networks, like small-worldness, highly connected hubs (together forming a rich club) and modularity, are already established at birth, they are thought to mature across childhood and adolescence (Vértes \& Bullmore, 2015;

Wierenga et al., 2018). Few longitudinal studies have so far been performed, but one such study found that the efficiency of structural networks as measured from DTI changes in a non-linear fashion from late childhood to early adulthood, and that development of network efficiency is related to intelligence (Koenis et al., 2017).

\section{Conclusion}

The human brain undergoes considerable changes in structure across the first two decades of life. Cortical grey matter increases into childhood, and decreases steadily across adolescence before stabilizing in the early twenties, whereas white matter increases. The cortex thins around $1 \%$ annually throughout the second decade of life, and surface area decreases at approximately half 
this rate. Crucially, cortical and subcortical changes do not proceed uniformly, but rather there are regional differences in timing and tempo, with a general trend for posterior regions to develop earlier than anterior regions of the brain. White matter microstructure also continues to change into the third decade of life, with frontotemporal connections developing more slowly than other tracts. Both morphometric properties and diffusion measures derived from MRI cannot currently be mapped to specific cellular processes. Our understanding of the underlying mechanisms driving structural changes in the brain will continue to improve as new measures and approaches become more widely applied to longitudinal investigations. 


\section{References}

Alemán-Gómez, Y., Janssen, J., Schnack, H., Balaban, E., Pina-Camacho, L., Alfaro-Almagro, F., ... Desco, M. (2013). The human cerebral cortex flattens during adolescence. The Journal of Neuroscience, 33(38), 15004-15010. https://doi.org/10.1523/JNEUROSCI.1459-13.2013

Alexander-Bloch, A., Giedd, J. N., \& Bullmore, E. (2013). Imaging structural co-variance between human brain regions. Nature Reviews. Neuroscience, 14(5), 322-336. https://doi.org/10.1038/nrn3465

Alexander-Bloch, A., Raznahan, A., Bullmore, E., \& Giedd, J. N. (2013). The Convergence of Maturational Change and Structural Covariance in Human Cortical Networks. The Journal of Neuroscience, 33(7), 2889-2899. https://doi.org/10.1523/JNEUROSCI.3554-12.2013

Beaulieu, C. (2009). CHAPTER 6 - The Biological Basis of Diffusion Anisotropy. In Heidi JohansenBerg \& Timothy E.J. Behrens (Eds.), Diffusion MRI (pp. 105-126). San Diego: Academic Press.

Benes, F. (1989). Myelination of cortical-hippocampal relays during late adolescence. Schizophrenia Bulletin, 15(4), 585-593.

Benes, F., Turtle, M., Khan, Y., \& Farol, P. (1994). Myelination of a key relay zone in the hippocampal formation occurs in the human brain during childhood, adolescence, and adulthood. Archives of General Psychiatry, 51(6), 477-484.

Betzel, R. F., \& Bassett, D. S. (2017). Generative models for network neuroscience: prospects and promise. Journal of The Royal Society Interface, 14(136), 20170623. https://doi.org/10.1098/rsif.2017.0623

Bourgeois, jean-P., \& Rakic, P. (1993). Changes of synaptic density in the primary visual cortex of the macaque monkey from fetal to adult stage. The Journal of Neuroscience, 13(7), 2801-2820.

Chang, Y. S., Owen, J. P., Pojman, N. J., Thieu, T., Bukshpun, P., Wakahiro, M. L. J., ... Mukherjee, P. (2015). White Matter Changes of Neurite Density and Fiber Orientation Dispersion during Human Brain Maturation. PLOS ONE, 10(6), e0123656. https://doi.org/10.1371/journal.pone.0123656

Concha, L., Livy, D. J., Beaulieu, C., Wheatley, B. M., \& Gross, D. W. (2010). In vivo diffusion tensor imaging and histopathology of the fimbria-fornix in temporal lobe epilepsy. The Journal of Neuroscience, 30(3), 996-1002. https://doi.org/10.1523/JNEUROSCI.1619-09.2010

Fjell, A. M., Westlye, L. T., Amlien, I., Tamnes, C. K., Grydeland, H., Engvig, A., ... Walhovd, K. B. (2015). High-expanding cortical regions in human development and evolution are related to higher intellectual abilities. Cerebral Cortex, 25(1), 26-34. https://doi.org/10.1093/cercor/bht201

Geeraert, B. L., Lebel, R. M., Mah, A. C., Deoni, S. C., Alsop, D. C., Varma, G., \& Lebel, C. (2017). A comparison of inhomogeneous magnetization transfer, myelin volume fraction, and diffusion tensor imaging measures in healthy children. NeuroImage. https://doi.org/10.1016/j.neuroimage.2017.09.019

Genc, S., Malpas, C. B., Holland, S. K., Beare, R., \& Silk, T. J. (2017). Neurite density index is sensitive to age related differences in the developing brain. NeuroImage, 148(Supplement C), 373-380. https://doi.org/10.1016/j.neuroimage.2017.01.023

Geng, X., Gouttard, S., Sharma, A., Gu, H., Styner, M., Lin, W., ... Gilmore, J. H. (2012). Quantitative tract-based white matter development from birth to age 2years. NeuroImage, 61(3), 542-557. https://doi.org/10.1016/j.neuroimage.2012.03.057

Gilmore, J. H., Shi, F., Woolson, S. L., Knickmeyer, R. C., Short, S. J., Lin, W., ... Shen, D. (2012). Longitudinal development of cortical and subcortical gray matter from birth to 2 years. Cerebral Cortex, 22(11), 2478-2485. https://doi.org/10.1093/cercor/bhr327

Glasser, M. F., \& Van Essen, D. C. (2011). Mapping human cortical areas in vivo based on myelin content as revealed by T1- and T2-weighted MRI. The Journal of Neuroscience, 31(32), 1159711616. https://doi.org/10.1523/JNEUROSCI.2180-11.2011 
Goddings, A.-L., Mills, K. L., Clasen, L. S., Giedd, J. N., Viner, R. M., \& Blakemore, S.-J. (2014). The influence of puberty on subcortical brain development. NeuroImage, 88, 242-251. https://doi.org/10.1016/j.neuroimage.2013.09.073

Grydeland, H., Walhovd, K. B., Tamnes, C. K., Westlye, L. T., \& Fjell, A. M. (2013). Intracortical Myelin Links with Performance Variability across the Human Lifespan: Results from T1- and T2-Weighted MRI Myelin Mapping and Diffusion Tensor Imaging. The Journal of Neuroscience, 33(47), 18618-18630. https://doi.org/10.1523/JNEUROSCI.2811-13.2013

Hedman, A. M., van Haren, N. E. M., Schnack, H. G., Kahn, R. S., \& Hulshoff Pol, H. E. (2012). Human brain changes across the life span: a review of 56 longitudinal magnetic resonance imaging studies. Human Brain Mapping, 33(8), 1987-2002. https://doi.org/10.1002/hbm.21334

Herting, M. M., Johnson, C., Mills, K. L., Vijayakumar, N., Dennison, M., Liu, C., ... Tamnes, C. K. (2017). Development of subcortical volumes across adolescence in males and females: A multisample study of longitudinal changes. BioRxiv, 171389. https://doi.org/10.1101/171389

Herting, M. M., Johnson, C., Mills, K. L., Vijayakumar, N., Dennison, M., Liu, C., ... Tamnes, C. K. (2018). Development of subcortical volumes across adolescence in males and females: A multisample study of longitudinal changes. NeuroImage, 172, 194-205. https://doi.org/10.1016/j.neuroimage.2018.01.020

Herting, M. M., \& Sowell, E. R. (2017). Puberty and structural brain development in humans. Frontiers in Neuroendocrinology, 44, 122-137. https://doi.org/10.1016/j.yfrne.2016.12.003

Hill, J., Inder, T., Neil, J., Dierker, D., Harwell, J., \& Van Essen, D. (2010). Similar patterns of cortical expansion during human development and evolution. Proceedings of the National Academy of Sciences of the United States of America, 107(29), 13135-13140. https://doi.org/10.1073/pnas.1001229107

Jbabdi, S., \& Behrens, T. E. (2013). Long-range connectomics. Annals of the New York Academy of Sciences, 1305, 83-93. https://doi.org/10.1111/nyas.12271

Khundrakpam, B. S., Lewis, J. D., Reid, A., Karama, S., Zhao, L., Chouinard-Decorte, F., ... Brain Development Cooperative Group. (2017). Imaging structural covariance in the development of intelligence. NeuroImage, 144(Pt A), 227-240. https://doi.org/10.1016/j.neuroimage.2016.08.041

Koenis, M. M. G., Brouwer, R. M., Swagerman, S. C., van Soelen, I. L. C., Boomsma, D. I., \& Hulshoff Pol, H. E. (2017). Association between structural brain network efficiency and intelligence increases during adolescence. Human Brain Mapping. https://doi.org/10.1002/hbm.23885

Krogsrud, S. K., Fjell, A. M., Tamnes, C. K., Grydeland, H., Mork, L., Due-Tønnessen, P., ... Walhovd, K. B. (2016). Changes in white matter microstructure in the developing brain-A longitudinal diffusion tensor imaging study of children from 4 to 11years of age. NeuroImage, 124(Part A), 473-486. https://doi.org/10.1016/j.neuroimage.2015.09.017

Le Bihan, D., \& Johansen-Berg, H. (2012). Diffusion MRI at 25: exploring brain tissue structure and function. NeuroImage, 61(2), 324-341. https://doi.org/10.1016/j.neuroimage.2011.11.006

Lebel, C., \& Beaulieu, C. (2011). Longitudinal development of human brain wiring continues from childhood into adulthood. The Journal of Neuroscience, 31(30), 10937-10947. https://doi.org/10.1523/JNEUROSCI.5302-10.2011

Lebel, C., Gee, M., Camicioli, R., Wieler, M., Martin, W., \& Beaulieu, C. (2012). Diffusion tensor imaging of white matter tract evolution over the lifespan. NeuroImage, 60(1), 340-352. https://doi.org/10.1016/j.neuroimage.2011.11.094

Lebel, C., Walker, L., Leemans, A., Phillips, L., \& Beaulieu, C. (2008). Microstructural maturation of the human brain from childhood to adulthood. NeuroImage, 40(3), 1044-1055. https://doi.org/10.1016/j.neuroimage.2007.12.053

Lewis, J. D., Evans, A. C., \& Tohka, J. (2017). T1 white/gray contrast as a predictor of chronological age, and an index of cognitive performance. BioRxiv, 171892. https://doi.org/10.1101/171892

Mah, A., Geeraert, B., \& Lebel, C. (2017). Detailing neuroanatomical development in late childhood and early adolescence using NODDI. PLOS ONE, 12(8), e0182340.

https://doi.org/10.1371/journal.pone.0182340 
Maier-Hein, K. H., Neher, P. F., Houde, J.-C., Côté, M.-A., Garyfallidis, E., Zhong, J., ... Descoteaux, M. (2017). The challenge of mapping the human connectome based on diffusion tractography. Nature Communications, 8(1), 1349. https://doi.org/10.1038/s41467-017-01285-x

Mills, K. L., Goddings, A.-L., Herting, M. M., Meuwese, R., Blakemore, S.-J., Crone, E. A., ... Tamnes, C. K. (2016). Structural brain development between childhood and adulthood: Convergence across four longitudinal samples. NeuroImage, 141, 273-281. https://doi.org/10.1016/j.neuroimage.2016.07.044

Mutlu, A. K., Schneider, M., Debbané, M., Badoud, D., Eliez, S., \& Schaer, M. (2013). Sex differences in thickness, and folding developments throughout the cortex. NeuroImage, 82, 200-207. https://doi.org/10.1016/j.neuroimage.2013.05.076

Olson, I. R., Heide, R. J. V. D., Alm, K. H., \& Vyas, G. (2015). Development of the uncinate fasciculus: Implications for theory and developmental disorders. Developmental Cognitive Neuroscience, 14(Supplement C), 50-61. https://doi.org/10.1016/j.den.2015.06.003

Paus, T. (2010). Growth of white matter in the adolescent brain: myelin or axon? Brain and Cognition, 72(1), 26-35. https://doi.org/10.1016/j.bandc.2009.06.002

Paus, T. (2013). How environment and genes shape the adolescent brain. Hormones and Behavior. https://doi.org/10.1016/j.yhbeh.2013.04.004

Qiu, A., Mori, S., \& Miller, M. I. (2015). Diffusion tensor imaging for understanding brain development in early life. Annual Review of Psychology, 66, 853-876. https://doi.org/10.1146/annurev-psych010814-015340

Raznahan, A., Lerch, J. P., Lee, N., Greenstein, D., Wallace, G. L., Stockman, M., ... Giedd, J. N. (2011). Patterns of Coordinated Anatomical Change in Human Cortical Development: A Longitudinal Neuroimaging Study of Maturational Coupling. Neuron, 72(5), 873-884. https://doi.org/10.1016/j.neuron.2011.09.028

Raznahan, A., Shaw, P., Lalonde, F., Stockman, M., Wallace, G. L., Greenstein, D., ... Giedd, J. N. (2011). How Does Your Cortex Grow? The Journal of Neuroscience, 31(19), 7174-7177. https://doi.org/10.1523/JNEUROSCI.0054-11.2011

Ritchie, S. J., Cox, S. R., Shen, X., Lombardo, M. V., Reus, L. M., Alloza, C., ... Deary, I. J. (2017). Sex Differences In The Adult Human Brain: Evidence From 5,216 UK Biobank Participants. BioRxiv, 123729. https://doi.org/10.1101/123729

Ruigrok, A. N. V., Salimi-Khorshidi, G., Lai, M.-C., Baron-Cohen, S., Lombardo, M. V., Tait, R. J., \& Suckling, J. (2014). A meta-analysis of sex differences in human brain structure. Neuroscience and Biobehavioral Reviews, 39(100), 34-50. https://doi.org/10.1016/j.neubiorev.2013.12.004

Schaer, M., Cuadra, M. B., Tamarit, L., Lazeyras, F., Eliez, S., \& Thiran, J. (2008). A Surface-Based Approach to Quantify Local Cortical Gyrification. IEEE Transactions on Medical Imaging, 27(2), 161-170. https://doi.org/10.1109/TMI.2007.903576

Schmierer, K., Wheeler-Kingshott, C. A. M., Boulby, P. A., Scaravilli, F., Altmann, D. R., Barker, G. J., ... Miller, D. H. (2007). Diffusion tensor imaging of post mortem multiple sclerosis brain. NeuroImage, 35(2), 467-477. https://doi.org/10.1016/j.neuroimage.2006.12.010

Simmonds, D., Hallquist, M. N., Asato, M., \& Luna, B. (2014). Developmental Stages and Sex Differences of White Matter and Behavioral Development through Adolescence: A Longitudinal Diffusion Tensor Imaging (DTI) Study. NeuroImage. https://doi.org/10.1016/j.neuroimage.2013.12.044

Song, S.-K., Yoshino, J., Le, T. Q., Lin, S.-J., Sun, S.-W., Cross, A. H., \& Armstrong, R. C. (2005). Demyelination increases radial diffusivity in corpus callosum of mouse brain. NeuroImage, 26(1), 132-140. https://doi.org/10.1016/j.neuroimage.2005.01.028

Tamnes, C. K., Herting, M. M., Goddings, A.-L., Meuwese, R., Blakemore, S.-J., Dahl, R. E., ... Mills, K. L. (2017). Development of the Cerebral Cortex across Adolescence: A Multisample Study of Inter-Related Longitudinal Changes in Cortical Volume, Surface Area, and Thickness. The Journal of Neuroscience, 37(12), 3402-3412. https://doi.org/10.1523/JNEUROSCI.3302-16.2017 
Tamnes, C. K., Roalf, D. R., Goddings, A.-L., \& Lebel, C. (2017). Diffusion MRI of white matter microstructure development in childhood and adolescence: Methods, challenges and progress. BioRxiv, 153924. https://doi.org/10.1101/153924

Tamnes, C. K., Walhovd, K. B., Dale, A. M., Østby, Y., Grydeland, H., Richardson, G., ... Fjell, A. M. (2013). Brain development and aging: Overlapping and unique patterns of change. NeuroImage, 68C, 63-74. https://doi.org/10.1016/j.neuroimage.2012.11.039

Vértes, P. E., \& Bullmore, E. T. (2015). Annual research review: Growth connectomics--the organization and reorganization of brain networks during normal and abnormal development. Journal of Child Psychology and Psychiatry, and Allied Disciplines, 56(3), 299-320. https://doi.org/10.1111/jcpp.12365

Vijayakumar, N., Allen, N. B., Youssef, G., Dennison, M., Yücel, M., Simmons, J. G., \& Whittle, S. (2016). Brain development during adolescence: A mixed-longitudinal investigation of cortical thickness, surface area, and volume. Human Brain Mapping. https://doi.org/10.1002/hbm.23154

Walhovd, K. B., Fjell, A. M., Giedd, J., Dale, A. M., \& Brown, T. T. (2016). Through Thick and Thin: a Need to Reconcile Contradictory Results on Trajectories in Human Cortical Development. Cerebral Cortex, bhv301. https://doi.org/10.1093/cercor/bhv301

Walhovd, K. B., Tamnes, C. K., Bjørnerud, A., Due-Tønnessen, P., Holland, D., Dale, A. M., \& Fjell, A. M. (2015). Maturation of Cortico-Subcortical Structural Networks-Segregation and Overlap of Medial Temporal and Fronto-Striatal Systems in Development. Cerebral Cortex, 25(7), 18351841. https://doi.org/10.1093/cercor/bht424

Wierenga, L. M., Langen, M., Ambrosino, S., van Dijk, S., Oranje, B., \& Durston, S. (2014). Typical development of basal ganglia, hippocampus, amygdala and cerebellum from age 7 to 24 . NeuroImage, 96, 67-72. https://doi.org/10.1016/j.neuroimage.2014.03.072

Wierenga, L. M., Langen, M., Oranje, B., \& Durston, S. (2014). Unique developmental trajectories of cortical thickness and surface area. NeuroImage, 87, 120-126. https://doi.org/10.1016/j.neuroimage.2013.11.010

Wierenga, L. M., Sexton, J. A., Laake, P., Giedd, J. N., \& Tamnes, C. K. (n.d.). A Key Characteristic of Sex Differences in the Developing Brain: Greater Variability in Brain Structure of Boys than Girls. Cerebral Cortex, 1-11. https://doi.org/10.1093/cercor/bhx154

Wierenga, L. M., van den Heuvel, M. P., Oranje, B., Giedd, J. N., Durston, S., Peper, J. S., ... The Pediatric Longitudinal Imaging, N., and Genetics Study. (2018). A multisample study of longitudinal changes in brain network architecture in 4-13-year-old children. Human Brain Mapping, 39(1), 157-170. https://doi.org/10.1002/hbm.23833

Yakovlev, P. A., \& Lecours, I. R. (1967). The myelogenetic cycles of regional maturation of the brain. In A. Minkowski (Ed.), Regional Development of the brain in early life. Oxford: Blackwell.

Zilles, K., Armstrong, E., Schleicher, A., \& Kretschmann, H.-J. (1988). The human pattern of gyrification in the cerebral cortex. Anatomy and Embryology, 179(2), 173-179.

https://doi.org/10.1007/BF00304699 

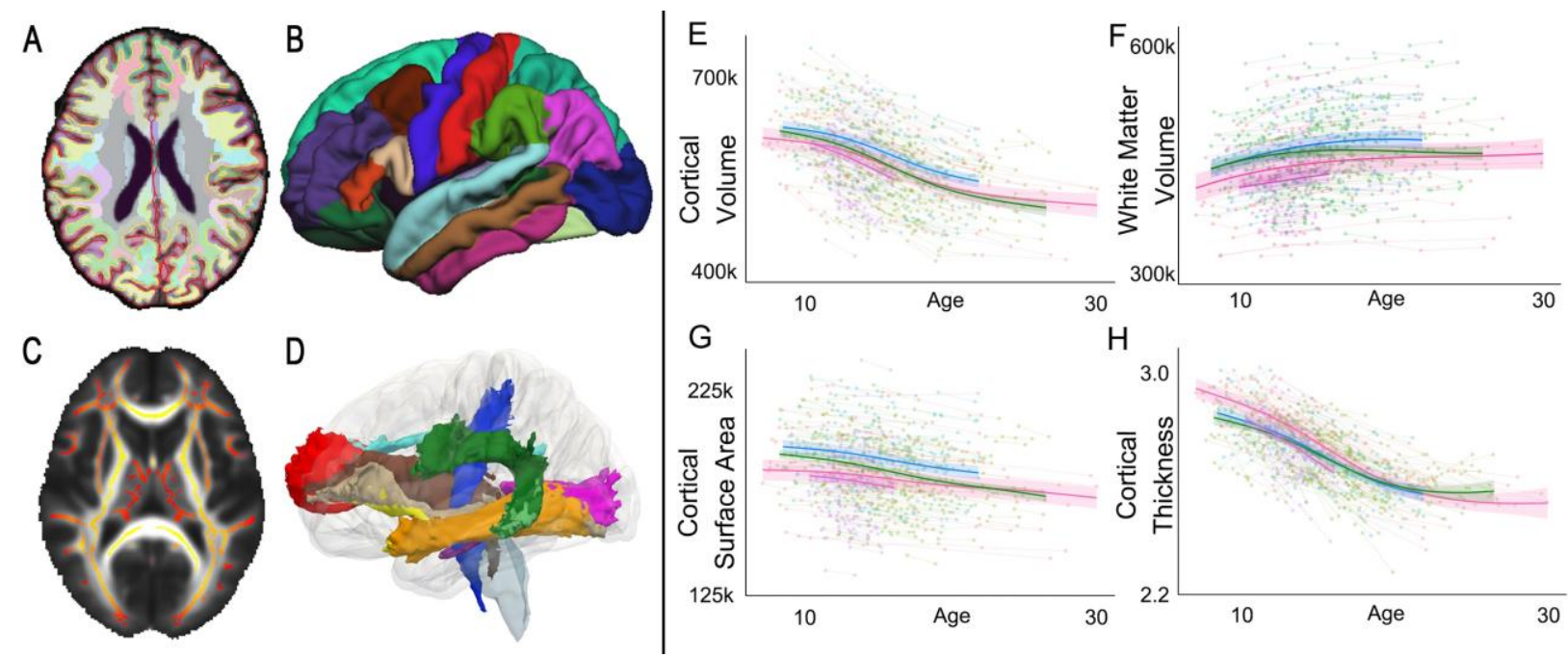

Fig. 1. Illustration of key MRI methods and findings discussed in this review. (A) Horizontal slice of T1 image showing a whole brain segmentation used for volumetric analyses and (B) a left lateral view of an averaged parcellated cerebral cortex used for surface-based analyses, both from FreeSurfer. (C) Horizontal slice of TBSS mean FA white matter skeleton overlaid on a mean FA map and (D) a left lateral view of a 3D rendering of probabilistic fiber tracts from the Mori atlas. Developmental trajectories for global cortical measures for cortical volume (E), total white matter volume $(F)$, cortical surface area $(G)$, and mean cortical thickness $(H)$. The colored lines represent the GAMM fitting while the lighter colored areas correspond to the $95 \%$ confidence intervals. Pink, CPB; purple, PIT; blue, NCD; green, BT. 